\title{
Designing for Photolurking
}

\author{
Haliyana Khalid \\ Lancaster University \\ Computing/Infolab21 \\ Lancaster, LA1 4YR, UK \\ +44 1524510349 \\ h.khalid@lancaster.ac.uk \\ http://www.lancs.ac.uk/postgrad/khalid/index.htm
}

\author{
Alan Dix \\ Lancaster University \\ Computing/Infolab21 \\ Lancaster, LA1 4YR, UK \\ +44 1524510319 \\ alan@hcibook.com \\ http://www.hcibook.com/alan/
}

\begin{abstract}
This paper describes our early work on design and development to support photolurking. Photolurking is browsing and looking at people's photographs without participating in discussion or addressing the owner of the photographs or photologs, whilst still discussing them in other avenues. We suggest several recommendations, including supporting ad-hoc instantaneous sharing, having remote and live discussion with groups of friends, and fostering collaborative experience. Having said that, the aim of this paper is not to propose an ideal application for supporting photolurking, but rather to provide an instance of how findings and analysis from ethnographic studies can feed into practical design.
\end{abstract}

\section{Categories and Subject Descriptors}

H.5.5 [Information Interfaces and Presentation]: HCI

\section{General Terms}

Design, Human Factors

\section{Keywords}

Photo sharing, photolurking, user experience, social networking

\section{INTRODUCTION}

There are many web-based public image sharing applications such as Flickr and Fotopages. In addition other social network applications, such as MySpace, Friendster, Facebook and blogs, offer photo sharing facilities as well. With this proliferation of opportunities to upload images, there are often too many photographs to look at and too many to choose from.

User experience in photo sharing has changed dramatically since the advent of digital photographs. Traditionally, personal photographs were shared among family members and friends, kept carefully in photo albums or stored in boxes and tins. Sharing was face to face or through the post. Art photography was shared through exhibition, magazine or books. However, just as technology has changed the nature of photography, so also has it changed the user experience of sharing.

This growing interest in public image-sharing applications and photologs was, for many, based on a desire to share experiences with remote friends and acquaintances, or to share skills and

(C)( Haliyana Khalid, Alan Dix, 2007

Published by the British Computer Society

Volume 2 Proceedings of the 21st BCS HCI Group Conference

HCI 2007, Lancaster University, 3-7 September 2007 photography techniques. However as they have developed and become more 'public' they also become a ways of showing your skills, your interests and yourself to the world.

Apart from photologgers (those who actually post images), visitors swamp into photologs for different reasons. Some are browsing to catch up with friends' and families' updates, some are searching for information like photography techniques, recipes and gossip, while others are just fulfilling their curiosity and boredom. Visitors and photologgers form an online community around the photolog; a new community that unites through photography. As one would expect, there are two types of visitors in online communities; active and passive. From our findings, active visitors frequently participated in the discussion column of a photolog, often acknowledgeable by the photolog owner. On the other hand, the passive visitors or as we named them, photolurkers $[7,9]$ do not offer any comments to the photolog yet often visited them.

Whilst the term 'photolurker' has a pejorative sound, but in fact our studies show this is not a negative experience. Photolurkers often communicate outside the photolog about the images they find within it and indeed photolurking is a valuable part of their social relationships. We therefore are looking at ways in which technology could be used to support this behaviour.

While studies of this sort often are regarded as having 'implications for design' [4], it is less common to see these implications worked through into actual design. In this paper we describe the early stages in the process of taking some of the findings of this research and pushing them into specific design concepts. These design concepts are currently being added into an existing web application Snip!t so that it can support discussion amongst photolurkers.

We will begin by briefly describing our ethnographic studies on the user experience in photologs that began in 2004, where we uncovered many interesting findings including the phenomenon of photolurking. This is followed by a few of the design recommendations which have arisen from this work, and which form the focus of this paper. We will then introduce Snip!t; an existing bookmarking tool, that allows one to snip a portion of the page content which can be stored and organized, but in addition can be passed to other web applications [3]. Methodologically this is important as real design is typically not ex nihilo, but instead builds on some existing platform, application or infrastructure. This base point often forms an additional constraint on the final product. We are using a scenario-based approach and we illustrate with a short scenario and how this is going to be integrated into Snip!t.

\section{BACKGROUND STUDY}

Our analysis based on user-experience studies on photologs since 2004 has focused attention on several interesting issues. 
Using various forms of ethnography, we have seen how personal memories of some people and other kinds of photographs are now freely shared on photologs. From our empirical studies, photos are shared on the application because of its mass-sharing capacity, responsibility to family and friends and to share techniques on photography and other skills. Apart from photologgers, photolog applications attract large number of viewers; both acquaintances and strangers. This combination of photologger and visitor has created the online community of the photolog. The visitors include silent visitor whom we described as 'photolurker' [7].

The word photolurker is derived from the word 'lurker' which means "one of the 'silent majority' in an electronic forum who posts occasionally or not at all but is known to read the postings of the group regularly" [8]. Our qualitative studies showed that our participants are more active photolurkers than photologgers. They visited people's photologs almost every day, often motivated or inspired by professional photographers or other people's photographs, which explains their frequent photolurking. We have suggested photolurking as term to describe this behaviour of browsing and looking at people's photographs without participating or acknowledging the owner of the photographs or photologs.

Personal attributes like shyness, being afraid to be recognised, lack of confidence with the language used and not having time to write are some of the reasons that prevent people from participating in discussions in photologs. However, our findings revealed that photolurkers do find other avenues to discuss the photographs. Encounters with photographs that trigger their interest are quickly considered, remembered and shared as this vignette from our studies demonstrates:

A group of friends are having breakfast together. As usual, they started to chat, catching up with stories like other friends would do. Then, one of them started to tell stories about a stranger and her photographs. Apparently, this stranger is no stranger to them, as most of them have seen her photos in her photolog. This group is reminiscing almost everything that they saw in the stranger photolog; her trip to Disneyland, her brother's convocation and who she's dating. Then the conversation flow to other people's photologs and gossips.

None of them has participated in the column discussion in the owner's photolog. However, discussions are made outside the photolog with a certain circle of friends in various ways such as face-to-face meetings (as in the above vignette), instant messaging or telephone conversation.

\section{DESIGN RECOMMENDATIONS}

As the focus of this paper is the movement from ethnography to design, we only present some specific findings here which suggest a potential for design. Briefly we need features that support user's desire for instantaneous sharing triggered by other people's photographs, and that allow remote discussion about those photographs privately with certain group of friends. The envisioned design also would benefit from synchronous communication and social interaction features. In the following, the ideas are broken down and further described.

\subsection{Supporting ad-hoc feeling to share}

Photolurking involves browsing and looking at people's photographs without participating openly in the discussion box or acknowledging the owner of the photographs. To date, there are thousands of photologs that display collections of photographs. This amount does not include social network application like MySpace and Facebook or weblog and newspapers that contains pictures.
Certain photographs elicit emotions to certain people. While browsing, people might encounter photographs or photologs that really interest them or reminded them of some occasions. Any encounters of photographs that trigger their interests are quickly thought, remembered and shared. Thus, photolurking deals with ad-hoc feelings and curiosity of people that further motivates shared experience. In our study;

"I was photolurking one night, when I spotted one celebrity's photolog. I've got very excited to see her photos and stories. I send message to all my friends in the block, and they rush to my room, and we look at the photologs together... We talk and then after that, everybody resume to their room."

This discussion was face to face and requires no explicit support. However, it demonstrates a user need to instantly share with others outside of the photolog itself to encourage further discussion and exploration. At the present, some photologs and other applications such as Friendster allow people to notify their friends about photos of their interest through email, but this communication channel does not support user need to share impulsively.

\subsection{Remote Discussion and Synchronous Communication}

One of the most common and enjoyable uses for photographs is to share stories about experiences, travels, friends and family [2]. However, with the advent of photolog, people don't just talk about their families and friends, but strangers as well. Interestingly, the life of a stranger (that could be seen through his photolog) can be an object of obsession and talked about to certain groups without them participating in the owner's photolog. From our findings, the discussion usually happened in face-to-face meetings, telephone and instant messaging.

Having a design that offers a remote discussion with synchronous communication channel immediately after seeing a photo would help to improve their shared experience. This also will help in communicating the context in which such photos are to be understood. Simultaneous discussions with selected friends encourage social interaction in the application.

Such an envisioned design would take advantage of instant messaging technology, but needs to be tied closely to the photos being discussed.

\subsection{Demonstrating social interaction}

While social networking sites share the basic purpose of online interaction and communication, specific goals and patterns of usage vary significantly across different services [5]. For example, MySpace allows for sharing profiles, LinkedIn for finding work, YouTube for video sharing and LiveJournal for sharing blogs.

Supporting photolurking that involves groups of users may benefit from creating a social network application. Unlike other social network application, the design will foster discussion about photographs and other articles on the Internet. This idea suggests many designs and user interface issues. Design challenges include ways to invite some friends to the discussion while also have the ability to save and to re-tell stories in the future. The design could also benefit from features allowing for invitation to the network, locating and discovering friends and connecting through different media.

These recommendations represent an instance of how the qualitative results study generated through ethnography can potentially 'inform' design. However, we would like to see these lead to far more specific design concepts. 


\section{INTEGRATING THE RECOMMENDATIONS INTO SNIP!T USING SCENARIOS}

One of the considerations in our designing for photolurking is that we are integrating our design strategies into an existing social bookmark tool called Snip!t. Snip!t was chosen as the authors have access to its developers and it already has some of the features that make it suitable as a basis.

As noted in the introduction real design rarely starts with a blank sheet. For similar reasons to a more commercial design process building a full application from scratch would not be effective in terms of time involved and by leveraging an existing platform we will be able to create a deployable system that can both act as a concept demonstrator for the results of existing studies and a technology probe [6] for future investigations.

\subsection{Existing features of Snip!t}

Snip!t is a web-based application that acts as a form of social bookmarking and also includes 'intelligent' features. A Snip!t user can select a portion of a web page and 'snip' it using a bookmarklet. The snipped portion together with the URL, title etc. are then saved in the web application and can be categorized, searched, and shared with others through email or public channels. In particular, the snipped part of the web page may include images (see Fig. 1), so this forms an initial way in which images can be selected form different photo sharing sites in order to be discussed.

In addition, Snip!t uses various patterns and heuristics in order to analyse the snipped area and web page. It looks for things like names, post codes, or telephone numbers and then suggests things which can be done with the matched areas; for example, a name might be looked-up in web-based people finders or an IP address checked in spam black-lists. The url of the page is also analysed so that, for example, the ISBN can be extracted from an Amazon page so that it is easy to use other services to find the book in a local library.

This feature means it would be possible to create patterns for popular photo-logs so that if the user snips a photo-log page this can trigger further rules to extract data, such as the title of the picture, from the page

\subsection{Scenario-based design}

Having Snip!t in mind, we are adopting scenario-based design methods to assist us with the process. This approach although perhaps the simplest design representation, is often considered one of the most flexible and powerful [1].

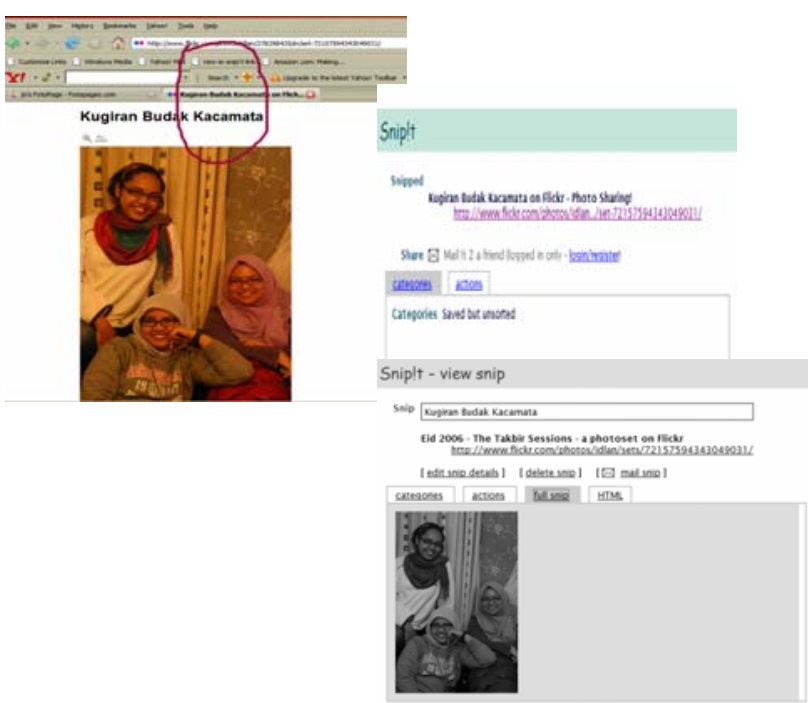

Figure 1. Snip!t used to snip an image

In this work, we are designing with a particular goal in mind supporting photolurking, especially remote discussion about other people's photos. However, we also need to create designs that can be potentially integrated with the existing application. The flexibility of scenarios is particularly important as a more generative approach (e.g. driving design form a task analysis) would run the risk of producing designs which whilst in theory good would not be deliverable in practice.

An example of short scenario to show an envisioned user task is as follows;

A is a frequent user of Snip!t. She uses Snip!t as a book marking tool and also to store interesting images that she notices while photolurking through photologs. She invites some of her friends to join Snip!t, and adds some friends who are already in Snip!t to join her network. One day, A found an image that really struck her emotion and would like to share it with her friends. She snips the picture to save it and sends an alert/message to some of her friends in her network to look at the image. A discussion box is supplied to allow them for discussion.

As is common, these scenarios are accompanied by sketches, and the design ideas. Critically the scenario above both sets itself into the context of Snip!t use (mentioned 4 times!) as well as photolurking. However, it is not limited to existing Snip!t features but adds functionality such as a network of friends and discussion areas.

One of the tensions in doing this work has been to create scenarios that are both (i) sufficiently unconstrained by the existing application that they suggest new design directions, but are also (ii) not so open that they are unimplementable! In practice, though we have lent towards an open design as the main aim of the scenarios is to explore detailed requirements not produce a final design.

\subsection{From scenarios to system}

As noted the scenarios suggest features not present in the exiting application as well as making use of current features. In addition in moving from the scenarios to a detailed design we looked extensions to that would be generally useful to Snip!t users as well as supporting photolurking.

The first element from the above scenario that is missing form the current systems is support for groups of friends. This is a common feature of such systems and has obvious benefits to the broader Snip!t users. Currently categories of snips can be either private to the user or public to everyone on the web. 
Adding networks of friends will mean that it is possible to share snips (of web pages as well as images) with selected people.

The other key element in the scenario is the discussion box. The first design idea, based on the above scenario, was to have an instant messenger style chat box attached to each snip. To allow slow discussions we decided this should be persistent and would be rather like comments on existing photologs except it would be private to a group.

However, further scenarios, themselves based on our previous studies of photo sharing, suggest that conversation would move form photo to photo, so the model of a conversation per photo would breakdown. From this, the conceptual model changed from one where chat was per picture, to one where pictures were moment-to-moment the topic of a chat.

Figure 2 shows a sketch of this with an area (1) for the current photo being discussed, a chat area (2) that includes both the typed contributions of the members of the chat group and also embedded thumbnails (3) when a new picture was introduced as the topic. Also at the bottom (4) is an unshared area for the user to collect images they would like to introduce into the chat.

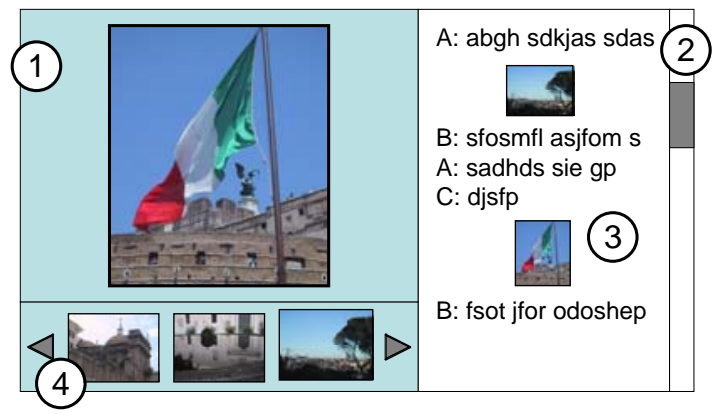

Figure 2. Potential photo chat

\section{CONCLUSIONS}

In this paper, we have described our initial work on the design and development of a tool to support photolurking. The design draws on our ethnographic studies of user experience in photologs. Building on Snip!t's existing ability to snip photographs and link to them from its web application, we are adding community and discussion facilities. These are each relatively simple, but together, we hope, will help answer users' ad hoc feelings and curiosity and the need for instant sharing and discussion. The flexibility of scenario-based methods have been crucial to balancing the goals of photolurking with the constraints of the existing application.

The choice of Snip!t was largely pragmatic based on our ability to influence its evolution. One alternative would be to alter an existing open-source image gallery. If we had done that, we would have been able to show how the design would fit into an existing photo-sharing site. However, actual deployment would have revealed little unless we had attracted a huge user base (unlikely!). In contrast, the Snip!t design builds off the presence of existing photo-sharing sites. Another alternative would be to use the API of one of the large sites to develop an application. However this would have been limited to a single site whereas Snip!t can allow discussion of multiple sites. It is interesting that these advantages of the proposed design were 'accidents' of the constraint to use Snip!t, but it has frequently been our observations that apparently arbitrary constraints lead to design innovation.

The ultimate aim of this phase of work is to deliver a deployable prototype. This will be given to subjects who we know already engage in both individual photolurking and also collective discussions, as described in section 3. We expect that the observations of logs and other observation techniques from protracted use will reveal other aspects of photolurking as well as further validate existing results.

\section{ACKNOWLEDGMENTS}

The study described in this paper was partially supported by Peel Trust Studentship, Lancaster University.

\section{REFERENCES}

[1] Benyon, D,. Turner, P. and Turner. S, Designing Interactive Systems. Pearson Education, 2005.

[2] Chaflen, R., Snapshot versions of life. Bowling Green State University Press. 1987.

[3] Dix, A., Catarci, T., Habegger, B., Ioannidis, Y., Kamaruddin, A., Katifori, A., Lepouras, G., Poggi, A., and Ramduny-Ellis, D.. Intelligent context- sensitive interaction on desktop and the web. In Proceedings of the International workshop in conjuction with AVI2006 on Context in advanced interfaces. 2006. Venice, Italy. ACM Press, New York, NY, 2000, 526-531.

[4] Dourish, P. 2006. Implications for design. In Proceedings of the SIGCHI Conference on Human Factors in Computing Systems (Montréal, Québec, Canada, April 22 - 27, 2006). R. Grinter, T. Rodden, P. Aoki, E. Cutrell, R. Jeffries, and G. Olson, Eds. CHI '06. ACM Press, New York, NY, 541-550.

[5] Gross, R. and Acquisti, A. Information revelation and privacy in online social networks. In Proceedings of the 2005ACM Workshop on Privacy in the Electronic Society WPES '05. 7 November, Alexandria, Virginia. 2005.

[6] Hutchinson, H., Mackay, W., Westerlund, B., Bederson, B. B., Druin, A., Plaisant, C., Beaudouin-Lafon, M., Conversy, S., Evans, H., Hansen, H., Roussel, N., and Eiderbäck, B. Technology probes: inspiring design for and with families. In Proceedings of the SIGCHI Conference on Human Factors in Computing Systems (Ft. Lauderdale, Florida, USA, April 05 - 10, 2003). CHI '03. ACM Press, New York, NY, 2003, 17-24.

[7] Khalid., H. and Dix, A.. From selective indulgence to engagement: exploratory studies on photolurking. In Extended Abstract of the British HCI 2006 Conference. Queen Mary, University of London, 11-15 September,2006.

[8] Nielsen, J. Participation Inequality: Encouraging More Users to Contribute Alertbox, October 9, 2006. www.useit.com/alertbox/participation_inequality.html

[9] WordSpy. Photolurker (definition and press references). Accessed 27 May 2007, available at: www.wordspy.com/words/photolurker.asp 